\title{
Decline in testosterone-dependent aggression of male rats following replacement of intact female cagemates with ovariectomized females
}

\author{
D. J. ALBERT, D. M. PETROVIC, and R. H. JONIK \\ University of British Columbia, Vancouver, British Columbia, Canada \\ and \\ M. L. WALSH \\ Simon Fraser University, Burnaby, British Columbia, Canada
}

\begin{abstract}
Male hooded rats were castrated and given subcutaneous implants of testosterone-filled Silastic tubes. Twenty-four hours later they were each housed with a female. At 7 and 14 days postoperatively, each male was observed for aggression toward an unfamiliar male intruder during a 15-min period. On Day 15 postoperatively, the female cagemate of each male was replaced with an unfamiliar intact female, an ovariectomized female, or a castrated male. Aggression toward an unfamiliar adult male was observed at 4,8 , and 12 weeks following the change of cagemate. Aggression remained at a high level in males whose female cagemates were exchanged for other intact females. Aggression declined significantly, but did not disappear, in males housed with an ovariectomized female or a castrated male. These results suggest that cohabitation with an intact female maintains hormone-dependent aggression in the male. The decline in aggression following removal of the intact female cagemate does not depend on a decline in serum testosterone concentration, since testosterone levels were maintained by subcutaneous testosterone implants.
\end{abstract}

The importance of testosterone for hormone-dependent aggression of the male rat is well documented. Testosterone must be present for the aggression to develop fully (Albert, Dyson, Walsh, \& Petrovic, 1988; Albert, Dyson, Walsh, \& Wong, 1988; Albert, Petrovic, \& Walsh, 1989; Albert, Walsh, Gorzalka, Siemens, \& Louie, 1986; Barfield, Busch, \& Wallen, 1972; Beeman, 1947; Christie \& Barfield, 1979; DeBold \& Miczek, 1984; Koolhaas, Schuurmann, \& Wiepkema, 1980; Schuurmann, 1980; Taylor, Haller, Rupich, \& Weiss, 1984). Removal of testosterone results in a decline in aggression (Albert et al., 1986; Barfield et al., 1972; Beeman, 1947; Christie \& Barfield, 1979; DeBold \& Miczek, 1984; Koolhaas et al., 1980; Schuurmann, 1980) and replacement causes the aggression to return (Albert et al., 1986; Beeman, 1947; Koolhaas et al., 1980; Schuurmann, 1980).

Less well known is the essential role of experiential events for hormone-dependent aggression. Group-housed males typically display little aggression toward an unfamiliar male (Albert, Dyson, Walsh, \& Petrovic, 1988;

The research in this article was supported by a grant from the Natural Sciences and Engineering Research Council of Canada. Reprint requests should be sent to D. J. Albert, Psychology Department, University of British Columbia, Vancouver, BC V6T 1Y7, Canada.

-Accepted by previous editor, Lynn Nadel
Barfield et al., 1972; Barnett, Evans, \& Stoddart, 1968; Barr, 1981; Flannelly \& Lore, 1977; Lore \& StipoFlaherty, 1984; Taylor et al., 1984). However, if males are allowed to cohabit with a female, the aggression is activated (Albert, Dyson, Walsh, \& Petrovic, 1988; Albert et al., 1986; Barnett et al., 1968; Barr, 1981; Blanchard, Fukunaga-Stinson, Takahashi, Flannelly, \& Blanchard, 1984; DeBold \& Miczek, 1984; Koolhaas et al., 1980; Taylor et al., 1984). The aggression is not usually directed toward the female cagemate but is displayed toward an unfamiliar male (Albert, Walsh, Zalys, \& Dyson, 1987; DeBold \& Miczek, 1984). Food competition or repeated exposure to unfamiliar males also activates hormone-dependent aggression (Albert, Dyson, Walsh, \& Petrovic, 1988; Albert, Dyson, Walsh, \& Wong, 1988; Lore \& Stipo-Flaherty, 1984; Zook \& Adams, 1975). Although some experiences that activate hormonedependent aggression are known to increase serum testosterone concentration temporarily (Flannelly \& Lore, 1977; Koolhaas et al., 1980; Schuurmann, 1980), the activation of aggression by experiential events does not depend on this increase (Albert, Dyson, Walsh, \& Petrovic, 1988; Albert, Dyson, Walsh, \& Wong, 1988; Taylor et al., 1984). The aggression is activated in males housed with females immediately following castration and in males that have been castrated and implanted with a stable testosterone supply (Albert, Dyson, Walsh, \& Gor- 
zalka, 1987; Albert, Dyson, Walsh, \& Petrovic, 1988; Albert, Dyson, Walsh, \& Wong, 1988).

Whether experiential events that activate testosteronedependent aggression must be continued in order to maintain the aggression has not been examined. If experiential events are necessary on a continuing basis, the aggressiveness should decline when the essential experiences are discontinued.

In the present experiment, we attempted to determine the effect of the continued presence of an intact female cagemate on the male's display of hormone-dependent aggression. Each male was allowed to cohabit with a female for a 2-week period. The female was then replaced with an unfamiliar intact female, an ovariectomized female, or a castrated male. Each male was tested for aggressiveness toward an unfamiliar male at 1-week intervals prior to changing cagemates, and at 4-week intervals afterward. Prior to being housed with a female, all males were castrated and implanted with testosterone-filled Silastic tubes to ensure that changes in behavior were not reflecting fluctuations in serum testosterone concentration.

\section{METHOD}

The subjects were male hooded rats $(300-350 \mathrm{~g})$ derived from stock obtained from Charles River Canada. Since weaning, they had been maintained in same-sex group cages with the same cagemates. Stable housing was used in order to minimize the activation of hormone-dependent aggression by repeated exposure to unfamiliar conspecifics (Lore \& Stipo-Flaherty, 1984). Females (250-300 g) were from the same strain. The animals were maintained on a normal 12:12-h light:dark cycle throughout the experiment. Behavioral observations were made during the last quarter of the light cycle.

\section{Surgery}

Males to be housed with females were castrated and then given bilateral subcutaneous implants of testosterone-filled Silastic tubes. Surgery was done according to standard surgical procedures, with sodium pentobarbital anesthesia. The subcutaneous implants (1.57 mm i.d.; $3.18 \mathrm{~mm}$ o.d., Dow Corning No. 602-285) had a center space $30 \mathrm{~mm}$ long filled with testosterone and were sealed at the ends with wood plugs and Silastic Type A sealant (Smith, Damassa, \& Davidson, 1977). They were subsequently implanted bilaterally at the level of the scapula. Testosterone-filled Silastic tubes with these dimensions are capable of supporting both sexual and aggressive behavior (Albert, Dyson, Walsh, \& Petrovic, 1988; Albert et al., 1986; Smith et al., 1977).

Postoperatively, the animals were housed with similarly treated cagemates for $24 \mathrm{~h}$. Each male was then housed with an intact female in a wood cage $50 \times 60 \times 20 \mathrm{~cm}$ high with dried corn cob chips on the floor, a hardware cloth top, and a Plexiglas front. Food and water were freely available.

\section{Behavioral Testing}

Males were observed for aggression toward an unfamiliar male intruder at 7 and 14 days following pairing with a female. Twentyfour hours after the second aggression test, the female cagemate was removed and replaced with an unfamiliar female, an ovariectomized female, or a castrated male. The males were assigned to groups in a manner that roughly balanced their composite aggression scores for the first two tests. The replacement females were similar in size to the original female cagemates. Each ovariectomized female had been subjected to surgery 1 week prior to being housed with the male. Each castrated male weighed about $50 \mathrm{~g}$ less than the resident male and had been castrated 2 weeks previously.

Aggression toward an unfamiliar male was reassessed at 4, 8, and 12 weeks following replacement of the original female cagemate. A longer intertest interval was used following pairing with the second cagemate, since repeated exposure to unfamiliar males can facilitate the expression of aggression even in the absence of a female cagemate (Albert, Dyson, Walsh, \& Petrovic, 1988; Lore \& Stipo-Flaherty, 1984).

Aggression toward an unfamiliar male was observed during a 15-min period on each test day. Fifteen minutes prior to the test, the resident cagemate was removed. The unfamiliar male intruder was introduced $15 \mathrm{~min}$ later. The males used as intruders weighed about $20 \%$ less than the resident males and were injected with a low dose of Diazepam ( $0.5 \mathrm{mg}$ i.p.) $15 \mathrm{~min}$ prior to the test. The use of smaller intruders was intended to facilitate the expression of aggression by the resident males. Diazepam produces more uniform intruder behavior by moderating defensiveness and decreasing freezing following an attack (Albert, Dyson, Walsh, \& Petrovic, 1988; Albert et al., 1986). Intruders were used not more than once a week and for not more than three tests.

Behaviors recorded during the aggression tests included attacks (lateral attacks, lunge attacks), bites, on-top, and piloerection. During a lateral attack, the attacking animal aligns itself parallel with the target animal, rises up on its toes, and pushes against the target animal with its flank or hind foot. A lunge attack was scored when the attacking animal jumped at the target animal and bit or pushed it. On-top was scored when the target animal lay on its back or side with the attacking animal holding it down or standing over it. A composite aggression score was calculated from the individual measures of aggression as follows: composite aggression = (number of attacks) +0.2 (attack duration in seconds) + (number of bites) +0.2 (on-top duration in seconds) + piloerection. Piloerection was scored as " 0 " if absent, " 2 " if partial or present for a part of the test session, and " 4 " if complete and present throughout the session. The composite aggression score has been found to be a more stable index of aggression than any individual measure (Albert, Dyson, Petrovic, \& Walsh, 1988; Albert, Dyson, et al., 1987; Albert, Dyson, Walsh, \& Petrovic, 1988; Albert et al., 1986; Albert, Walsh, et al., 1987).

Following behavioral testing, the resident males were killed. The testosterone tubes were retrieved and the castration confirmed.

\section{Statistical Analysis}

Each measure of aggression was subjected to a one-way analysis of variance with repeated measures. A Newman-Keuls Test was used to make individual comparisons for each test day. Piloerection scores were subjected to an overall chi-square contingency test.

\section{RESULTS}

\section{Composite Aggression Scores}

Differences between groups emerged following the change of cagemates [group, $F(2,24)=7.4, p<.01$; test day, $F(4,96)=7.0, p<.001$; linear interaction, $F(2,24)=12.3, p<.001$; see Figure 1]. Males housed with intact females $(N=10)$ maintained their levels of aggression toward the intruders at the monthly tests. Males whose intact female cagemates were exchanged for ovariectomized females $(N=10)$ or castrated males $(N=7)$ displayed levels of aggression that were significantly lower than those of males housed with intact females (Figure 1). 

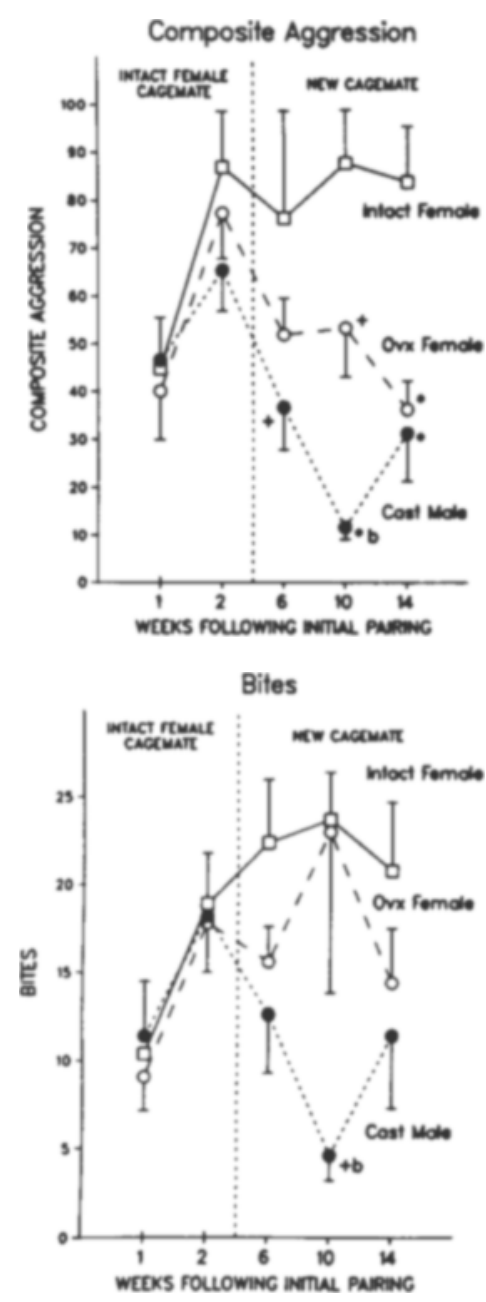
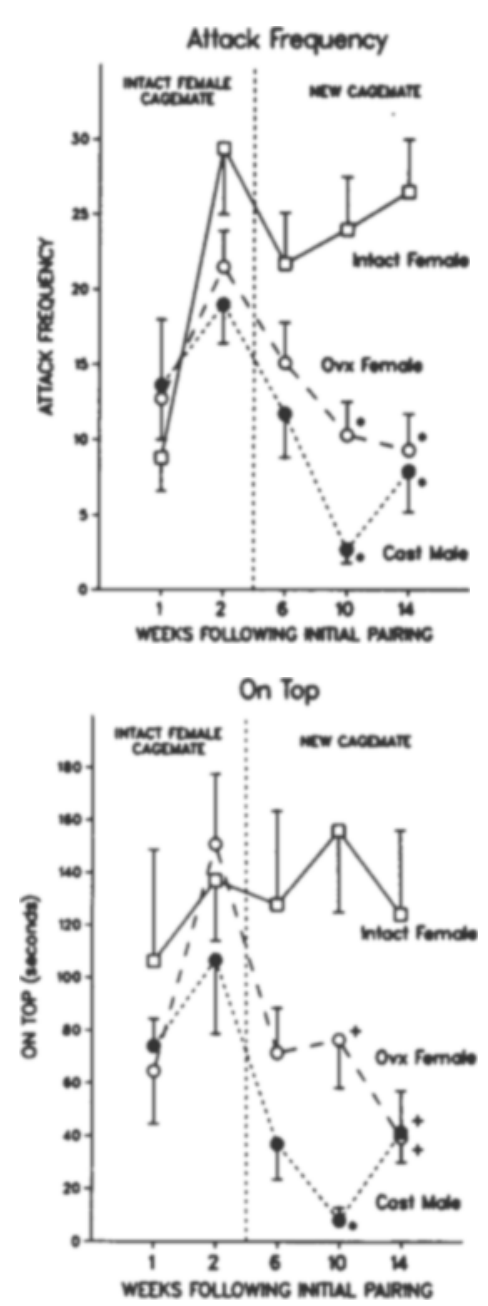
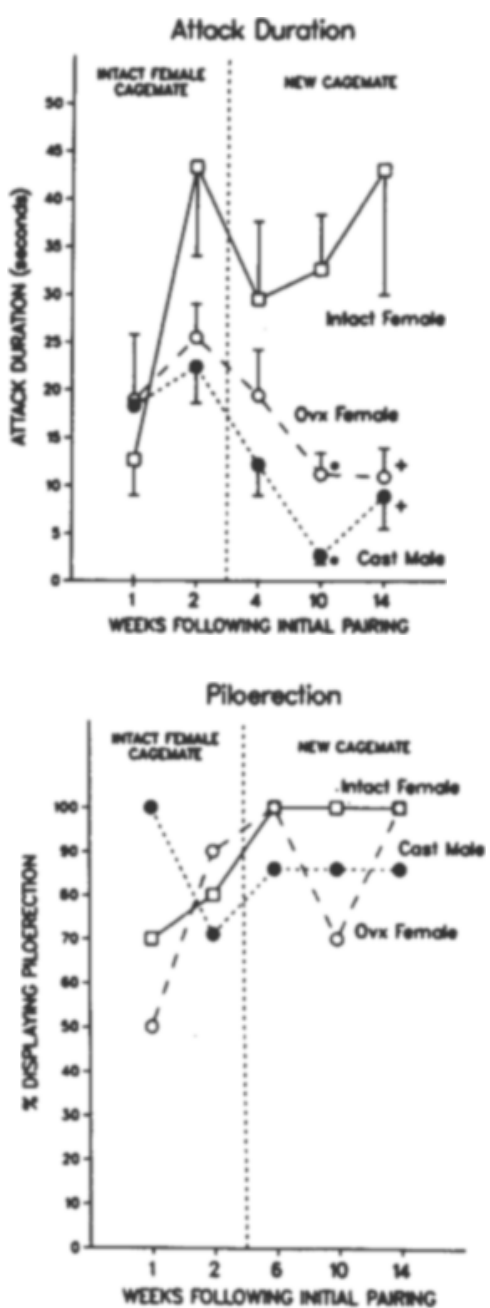

Figure 1. Measures of aggression toward an unfamiliar male. The vertical dotted line indicates the interval when the intact female cagemate was replaced with an unfamiliar intact female, an ovariectomized female (Ovx Female), or a castrated male (Cast Male). Filoerection scores represent the percentage of animals displaying either partial or full piloerection. Error bars represent SEMs. Significant differences between the aggression of males housed with intact females and each of the other groups: $p<.01 ;+p<.05$. Significant differences in aggression by males housed with an ovariectomized female and those housed with a castrated male: $b=p<.05$.

There were no significant differences between groups at either test prior to the change of cagemate (all $p s>.30$ ).

Aggression did not disappear following the change of cagemates. At the final test, 3 months after removal of the intact female, males cohabiting with either ovariectomized females or castrated males displayed levels of aggression similar to those displayed after cohabitation with intact females for 1 week.

Castrated males replacing intact females were frequently attacked shortly after being placed in the colony. These attacks usually disappeared within 1-2 days. In one instance, the attacks reappeared during the final 6 weeks and took the form of repeated bites at the paws and tail. Removing the castrated male from the cage for 1 night would stop the attacks for several days. There were no concerted attacks on newly introduced intact or ovariectomized females.

\section{Individual Measures of Aggression}

Attack frequency, attack duration, and on-top followed the pattern of change seen in the composite aggression score [group, all $F_{\mathrm{s}}(2,24)>5.7$, all $p s<.01$; test day, all $F_{\mathrm{s}}(4,96)>3.0$, all $p \mathrm{~s}<.02$; linear interaction, all $F \mathrm{~s}(2,24)>3.5$, all $p \mathrm{~s}<.05$ ]. In each case, there were no differences between groups during the first two tests (all $p s>.30$; see Figure 1). Following the change of cagemate, the males housed with ovariectomized females or castrated males showed significant declines relative to the control group (Figure 1). With each measure, the aggression clearly did not disappear over the 3-month test period.

Bite scores also followed the pattern of change seen in the composite aggression scores [Group, $F(2,24)=2.2$, $p>.10$; test day, $F(4,96)=3.8, p<.01$; linear interaction, $F(2,24)=4.4, p<.02$ ]. Bite scores of males 
housed with intact females remained remarkably consistent following the change of cagemate (Figure 1). However, although males housed with ovariectomized females or castrated males showed consistently lower bite scores than did the males housed with intact females, large within-groups variability resulted in few statistically significant differences on individual test days (Figure 1).

The proportion of animals in each group displaying either partial or full piloerection did not differ significantly between groups $\left(\chi^{2}=26, p>.10\right)$. The frequency of piloerection in all groups remained at about the level seen in the test immediately prior to the change of cagemate (Figure 1).

\section{DISCUSSION}

Experiential events activate hormone-dependent aggression in male rats. Prior to cohabiting with an intact female or experiencing a competitive situation, most males do not attack an unfamiliar male (Albert, Dyson, Walsh, \& Petrovic, 1988; Barnett et al., 1968; Barr, 1981; Flannelly \& Lore, 1977; Lore \& Stipo-Flaherty, 1984; Taylor et al., 1984). Following exposure to one of these situations, virtually all male rats respond to an unfamiliar male with aggression (Albert, Dyson, Walsh, \& Petrovic, 1988; Albert et al., 1986; Barnett et al., 1968; Barr, 1981; Blanchard et al., 1984; DeBold \& Miczek, 1984; Koolhaas et al., 1980; Taylor et al., 1984). The activation of aggression is not dependent on an increase in serum testosterone concentration, since it develops in males whose serum testosterone concentration has been stabilized by replacing testicular testosterone with subcutaneous testosterone implants (Albert, Dyson, et al., 1987; Albert, Dyson, Walsh, \& Petrovic, 1988; Albert, Dyson, Walsh, \& Wong, 1988; Albert et al., 1986).

Experiential events also appear to maintain hormonedependent aggression. Aggression toward an unfamiliar male persisted at a high level only in animals that continued to cohabit with an intact female (Figure 1). A decline in aggression was seen in males whose intact female cagemate was exchanged for an ovariectomized female or a castrated male. Notably, the aggression did not disappear during the 3-month test period. In fact, the frequency of developing piloerection did not decline significantly, indicating that males housed with ovariectomized females or castrated males continued to recognize the intruder as foreign and to respond to it with the initial element of aggression. The persistence of piloerection is not entirely unexpected, since piloerection by a male housed with an intact female has been reported to be comparable to that of a male housed with another male, even though the attack scores of these animals are significantly different (Albert, Dyson, Walsh, \& Petrovic, 1988).

Males housed with ovariectomized females or castrated males tended to look as though they were about to attack the intruder. Although attacks usually did not occur, some behaviors, such as running away by the target animal, appeared to increase the probability of an attack. Aggres- sion might have declined more completely if males without access to intact females were intermittently challenged by male cagemates with access to intact females. This would parallel conditions under which hormone-dependent aggression has been observed to decline following testosterone removal (Albert et al., 1986).

Balancing groups by composite aggression scores resulted in a substantial imbalance in the attack frequency and attack duration scores for the first two weekly tests (Figure 1). Although the difference between groups during the first two tests was not significant on either measure, it was in the direction that would occur following the change of cagemates. Previous experiments have suggested that differences as large as those present in these tests frequently occur in unselected groups during the first two weekly tests. These differences do not foreshadow the subsequent appearance of significant differences in aggression such as those seen in Figure 1 (see, e.g., Albert, Dyson, Walsh, \& Petrovic, 1988, Figure 1).

The absence of heterosexual activity is presumably responsible for the decline in aggression in males housed with ovariectomized females or castrated males. Sexual activity enhances the aggressiveness of males (Flannelly, Blanchard, Muraoka, \& Flannelly, 1982; Taylor et al., 1984). Inaccessible estrous females can also enhance aggression of males (Taylor et al., 1984). In the present experiment, estrous females in the vicinity of males housed with ovariectomized females or castrated males may have attenuated the decline of aggression by those males. The decline in male hormone-dependent aggression following removal of an intact female cagemate was not a reflection of a decrease in serum testosterone concentration. Serum testosterone concentration can be inferred to have been relatively stable, since all males had been castrated and implanted with testosterone-filled Silastic tubes.

Evidence that experiential events maintain aggressiveness in male rats parallels observations made on females. Hormone-dependent aggression in the female is fostered by competitive experience and cohabitation with a sterile male, as well as by pregnancy, parturition, and lactation (Albert, Dyson, Petrovic, \& Walsh, 1988; Erskine, Barfield, \& Goldman, 1978; Flannelly \& Flannelly, 1987; Mayer, Reisbick, Siegel, \& Rosenblatt, 1987). Aggressiveness fluctuates widely in the case of pregnancy, parturition, and lactation (Albert, Dyson, Petrovic, \& Walsh, 1988; Flannelly \& Flannelly, 1987; Flannelly, Flannelly, \& Lore, 1986; Mayer, Carter, et al., 1987; Mayer, Reisbick, et al., 1987). It declines as lactation comes to an end (Albert, Dyson, Petrovic, \& Walsh, 1988; Mayer, Reisbick, et al., 1987), but it can actually disappear if the pups are removed for several hours (Erskine et al., 1978). There have been no comparable observations that the stability of aggressiveness in males depended on experiential events. The present experiment provides some initial evidence regarding this issue.

To summarize, aggression of castrated male rats with testosterone implants increased following cohabitation with females. When the female cagemate was exchanged 
for an unfamiliar intact female, aggression toward an unfamiliar intruder remained unchanged. However, when the female cagemate was exchanged for an ovariectomized female or a castrated male, aggression declined. The decline in aggression occurred even though serum testosterone concentration was maintained by subcutaneous testosterone implants. It may be inferred that aggression toward an unfamiliar male is maintained as well as activated by the presence of an intact female.

\section{REFERENCES}

Albert, D. J., Dyson, E. M., Petrovic, D. M., \& Walsh, M. L. (1988). Activation of aggression in female rats by normal males and by castrated males with testosterone implants. Physiology \& Behavior, 44, 9-13.

Albert, D. J., Dyson, E. M., Walsh, M. L., Gorzalka, B. B. (1987). Intermale social aggression in rats: Suppression by medial hypothalamic lesions independently of enhanced defensiveness or decreased testicular testosterone. Physiology \& Behavior, 39, 693-698.

Albert, D. J., Dyson, E. M., Walsh, M. L., a Petrovic, D. M. (1988). Cohabitation with a female activates testosterone-dependent social aggression in male rats independently of changes in serum testosterone concentration. Physiology \& Behavior, 44, 735-740.

Albert, D. J., Dyson, E. M., Walsh, M. L., \& Wong, R. (1988). Defensive aggression and testosterone-dependent intermale social aggression are each elicited by food competition. Physiology \& Behavior, 43, 21-28.

Albert, D. J., Petrovic, D. M., \& Walsh, M. L. (1989). Ovariectomy attenuates aggression by female rats cohabiting with sexually active sterile males. Physiology \& Behavior, 45, 225-228.

Albert, D. J., Walsh, M. L., Gorzalka, B. B., Siemens, Y., \& LouIE, H. (1986). Testosterone removal in rats results in a decrease in social aggression and a loss of social dominance. Physiology \& Behavior, 36, 401-407.

Albert, D. J., WAlsh, M. L., ZAlys, C., \& Dyson, E. M. (1987). Maternal aggression and intermale social aggression: A behavioral comparison. Behavioural Processes, 14, 267-275.

Barfield, R. J., Busch, D. E., \& Wallen, K. (1972). Gonadal influences on agonistic behavior in the male domesticated rat. Hormones \& Behavior, 3, 247-259.

Barnett, S. A., Evans, C. S., \& Stoddart, R. C. (1968). Influence of females upon conflict among wild rats. Joumal of Zoology, 154, 391-396.

BARR, G. A. (1981). Effects of different housing conditions on intraspecies fighting between male Long-Evans hooded rats. Physiology \& Behavior, 27, 1041-1044.

Beeman, E. A. (1947). The effect of male hormone on aggressive behavior in mice. Physiological Zoology, 20, 393-405.
Blanchard, D. C., Fukunaga-Stinson, C., Takahashi, L. K., Flannelly, K. J., Blanchard, R. J. (1984). Dominance and aggression in social groups of male and female rats. Behavioural Processes, 9, 31-48.

Christie, M. J., B BArfield, R. J. (1979). Effects of castration and home cage residence on aggressive behavior in rats. Hormones \& Behavior, 13, 85-91.

DeBold, J. F., Miczex, K. A. (1984). Aggression persists after ovariectomy in female rats. Hormones \& Behavior, 18, 177-190.

Erskine, M. S., Barfield, R. J., Goldman, B. D. (1978). Intraspecific fighting during late pregnancy and lactation in rats and effects of litter removal. Behavioral Biology, 23, 206-218.

Flannelly, K. J., Blanchard, R. J., Muraoka, M. Y., a FlanNELLY, L. (1982). Copulation increases offensive attack in male rats. Physiology \& Behavior, 29, 381-385.

Flannelly, K. J., \& Flannelly, L. (1987). Time course of postpartum aggression in rats (Ratmus norvegicus). Joumal of Comparative \& Physiological Psychology, 101, 101-103.

Flannelly, K. J., Flannelly, L., Lore, R. K. (1986). Post partum aggression against intruding male conspecifics in Sprague-Dawley rats. Behavioural Processes, 13, 279-386.

Flannelly, K. J., LoRE, R. K. (1977). The influence of females upon aggression in domesticated male rats (Rattus norvegicus). Animal Behaviour, 25, 654-659.

Koolhans, J. M., Schuurmann, T., Wiepkema, P. R. (1980). The organization of intraspecific agonistic behaviour in the rat. Progress in Neurobiology, 14, 247-268.

Lore, R. K., Stipo-Flaherty, A. (1984). Postweaning social experience and adult aggression in rats. Physiology \& Behavior, 33, 571-574.

Mayer, A. D., Carter, L., Jorge, W. A., Mota, M. J., Tannu, S., - Rosenblatt, J. S. (1987). Mammary stimulation and maternal aggression in rodents: Thelectomy fails to reduce pre- or postpartum aggression in rats. Hormones \& Behavior, 21, 501-510.

Mayer, A. D., Reisbick, S., Siege., H. I., a Rosenblatt, J. S. (1987) Maternal aggression in rats: Changes over pregnancy and lactation in Sprague-Dawley strain. Aggressive Behavior, 13, $29-43$.

SChUURMANN, T. (1980). Hormonal correlates of agonistic behavior in adult male rats. Progress in Brain Research, 53, 415-420.

Smith, E. R., Damassa, D. A., \& Davidson, J. M. (1977). Hormone administration: Peripheral and intracranial implants. In R. D. Meyers (Ed.), Methods in psychobiology (Vol. 3, pp. 259-279). New York: Academic Press.

Taylor, G. T., Haller, J., Rupich, R., Weiss, J. (1984). Testicular hormones and intermale aggressive behaviour in the presence of a female rat. Journal of Endocrinology, 100, 315-321.

Zook, J. M., ADAMs, D. B. (1975). Competitive fighting in the rat. Journal of Comparative \& Physiological Psychology, 88, 418-423.

(Manuscript received April 24, 1989; revision accepted for publication January 17,1990 .) 